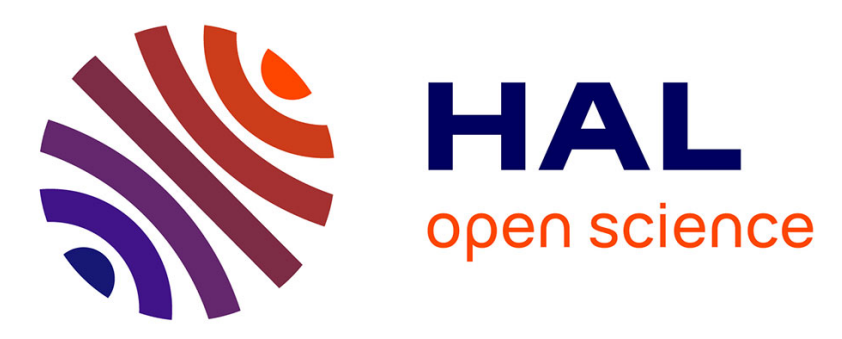

\title{
Modal Superimposition for Multi-fingers Variable Friction Tactile Device
}

Sofiane Ghenna, Christophe Giraud-Audine, Frédéric Giraud, Michel Amberg, Betty Semail

\section{- To cite this version:}

Sofiane Ghenna, Christophe Giraud-Audine, Frédéric Giraud, Michel Amberg, Betty Semail. Modal Superimposition for Multi-fingers Variable Friction Tactile Device. Haptics: Perception, Devices, Control, and Applications , 9774, , pp.521-530, 2016, 10.1007/978-3-319-42321-0_49 . hal-01360917

\section{HAL Id: hal-01360917 https://inria.hal.science/hal-01360917}

Submitted on 6 Sep 2016

HAL is a multi-disciplinary open access archive for the deposit and dissemination of scientific research documents, whether they are published or not. The documents may come from teaching and research institutions in France or abroad, or from public or private research centers.
L'archive ouverte pluridisciplinaire HAL, est destinée au dépôt et à la diffusion de documents scientifiques de niveau recherche, publiés ou non, émanant des établissements d'enseignement et de recherche français ou étrangers, des laboratoires publics ou privés. 


\title{
Modal superimposition for multi-fingers Variable Friction tactile Device
}

\author{
Sofiane Ghenna, Christophe Giraud-Audine, Frederic Giraud, Michel Amberg, \\ and Betty Semail \\ Univ. Lille, Centrale Lille, Arts et Metiers ParisTech, HEI, HeSam, EA 2697 - L2EP - \\ Laboratoire dElectrotechnique et dElectronique de Puissance, F-59000 Lille, France \\ name.surname@univ-lille1.fr, christophe.giraud-audine@ensam.eu
}

\begin{abstract}
In this study, we develop and implement a method for superimposing two vibration modes in order to produce different tactile stimuli on two fingers located in different positions. The tactile stimulation is based on the squeeze film effect which decreases the friction between a fingertip and a vibrating plate.

Experimental test have been conducted on a 1D tactile device. They show that it is possible to continuously control the friction on two fingers moving independently. Then, we developed the design of a $2 \mathrm{D}$ device based on the same principle, which gives rise to the design of a two-fingers tactile display. Evaluations were conducted using a modal analysis with experimental validation.
\end{abstract}

Keywords: ultrasonic, vibration modes, tactile stimulation, multi-touch

\section{Introduction}

The Variable Friction Devices (VFD) modulate the friction between a user's fingertip and a surface to create tactile stimulation. Electrostatic forces can be used to attract the finger and thus to increase the friction [2]. Friction reduction based tactile devices use an ultrasonic vibration to modify the contact mechanisms between a user's fingertip and a vibrating plate [10]. Many implementations of this principle have been proposed, to create $2 \mathrm{D}$ tactile displays. For instance [3] uses a circular patch glued on the screen of a tactile display. In order to free the touched surface of non-transparent piezo-electric material, [5] proposes to produce the vibration with exciters glued at the periphery of the display. Finally, recent optimization in the design helps to improve the efficiency and limit the amount of power requested by the tactile stimulator [11], thus allowing implementation into handheld devices.

These implementations are using a single Rayleigh vibration mode [7]. In order to prevent a user's finger from crossing or feeling a stationary nodal lines, the distance between two nodal lines of vibration - that is half a wavelength - should be comparable with the finger 's contact length. In practice, for a light touch exploration [1], this leads to a maximum length of $12 \mathrm{~mm}$. An other 
approach consists in switching between two vibration modes, the nodal lines of which have orthogonal directions [9].

These approaches however are limited to a single touch interaction. Indeed, they do not allow to precisely control the vibration amplitudes at two or more different positions simultaneously. To cope with this issue, [4] proposed to superimpose two vibration modes, and to control the vibration amplitude of each mode according to the position of two fingertips. This principle take advantages of modal superimposition by using two modes which half wavelength is higher than $12 \mathrm{~mm}$.

So far, the principle has been validated in static operation and for 1D tactile display. In this paper, we further validate the proposal in the case of tracking two fingers, and we extend the principle to $2 \mathrm{D}$ displays. In the first part, we briefly recall the principles of the modal superimposition. We then present the validation with a tracking of two fingers with a psychophysical study. In the last part, we show evidence that the approach is also valid for $2 \mathrm{D}$.

\section{Presentation of the multimodal approach}

Vibrations of a plate can be written as a combination of an infinite number of modes, determined by the equilibrium equation [7]:

$$
D \nabla^{4} w(x, y, t)+\rho h \frac{\partial^{2} w(x, y, t)}{\partial t^{2}}=0
$$

where $w(x, y, t)$ is the flexural vibration, $\rho, h$ and $\partial$ are respectively the density and the thickness of plate and the partial derivative. $D$ is the flexural rigidity of the plate in bending defined by

$$
D=\frac{E h^{3}}{12\left(1-\mu^{2}\right)}
$$

$E$ and $\mu$ are the Young module and poisson's ratio of the plate.

together with the kinematic boundary conditions.

For a steady state and monochromatic vibration at angular frequency $\omega$, one can write the solution using complex notations using $\underline{w}(x, y, t)=\underline{W}(x, y, t) e^{j \omega t}$ as follows:

$$
\underline{W}(x, y, t)=\sum_{n=1}^{\infty} \underline{W}_{n}(t) \phi_{n}(x, y)
$$

with $\phi_{n}(x, y)$ are the modal shapes, in the sequel, $\underline{W}_{n}$ is called the vibration amplitude of mode $n$.

Hence, theoretically, the vibration shape $w(x, y, t)$ can be controlled at the condition that the vibration amplitude of each mode $\underline{W}_{n}$ is controlled too.

Therefore, if we consider $m$ different positions on the plate namely $\left(x_{i}, y_{i}\right)$ where $i=\{1 . . m\}$, where the deformation must be controlled to render different tactile stimulations, we can select at least $p \geq m$ modes of vibration to write the problem as follows: 


$$
\left[\begin{array}{c}
\underline{W}\left(x_{1}, y_{1}\right) \\
\underline{W}\left(x_{2}, y_{2}\right) \\
\vdots \\
\underline{W}\left(x_{m}, y_{m}\right)
\end{array}\right]=\left[\begin{array}{cccc}
\phi_{1}\left(x_{1}, y_{1}\right) & \phi_{2}\left(x_{1}, y_{1}\right) & \cdots & \phi_{p}\left(x_{1}, y_{1}\right) \\
\phi_{1}\left(x_{2}, y_{2}\right) & \phi_{2}\left(x_{2}, y_{2}\right) & \cdots & \phi_{p}\left(x_{2}, y_{2}\right) \\
\vdots & \vdots & \ddots & \vdots \\
\phi_{1}\left(x_{m}, y_{m}\right) & \phi_{2}\left(x_{m}, y_{m}\right) & \cdots & \phi_{p}\left(x_{m}, y_{m}\right)
\end{array}\right]\left[\begin{array}{c}
\underline{W}_{1} \\
\underline{W}_{2} \\
\vdots \\
\underline{W}_{p}
\end{array}\right]
$$

This can be written in matrix form as:

$$
\left[W_{f}\right]=\left[\Phi_{f}\right] \cdot\left[W_{M}\right]
$$

Where $\left[\Phi_{f}\right]$ is a $m \times p$ matrix. It is possible to find a vector of modal deformation amplitude $\left[W_{M}\right]$ which can be set in order to obtain the required deformation of the plate at the positions of the fingers $\left[W_{f}\right]$ :

- if $m=p,\left[W_{M}\right]=\left[\Phi_{f}\right]^{-1} \cdot\left[W_{f}\right]$ if $\operatorname{det}\left[\Phi_{f}\right] \neq 0[4]$,

- if $p>m$, a solution can be found either using the pseudo inverse of $\left[\Phi_{f}\right]$ which is equivalent to the least square approximation, or by using more elaborated optimization to introduce technological constraints for example.

In the rest of the paper, we consider two fingers and two vibration modes. For the specific case of $2 D$ and 2-fingers VFD, they are denoted by $\phi_{k, X}$ and $\phi_{k, Y}$, where $k$ is an integer. For the purpose of demonstration, and without loss of generality, we can approximate the modal shapes by simplified equations as follows :

$$
\phi_{k, X}(x, y)=W_{X} \sin \left(\lambda_{k} x\right) \quad \text { and } \quad \phi_{k, Y}(x, y)=W_{Y} \sin \left(\lambda_{k} y\right)
$$

with $\lambda_{k}=(2 k+1) \frac{\pi}{2}$. Therefore, the problem of controlling the amplitudes under the user's fingers at positions $\left(x_{1}, y_{1}\right)$ and $\left(x_{2}, y_{2}\right)$ is stated by :

$$
\left\{\begin{array}{l}
W_{X} \sin \left(\lambda_{k} x_{1}\right)+W_{Y} \sin \left(\lambda_{k} y_{1}\right)=W_{1} \\
W_{X} \sin \left(\lambda_{k} x_{2}\right)+W_{Y} \sin \left(\lambda_{k} y_{2}\right)=W_{2}
\end{array}\right.
$$

where the amplitude of the modes $X$ and $Y$ are denoted $W_{X}$ and $W_{Y}$ respectively. The problem has a solution for the modes amplitude $W_{1}$ and $W_{2}$, as long as the determinant is not nil, which gives rise to the condition :

$$
\sin \left(\lambda_{k} x_{1}\right) \sin \left(\lambda_{k} y_{2}\right)-\sin \left(\lambda_{k} x_{2}\right) \sin \left(\lambda_{k} y_{1}\right) \neq 0
$$

At the positions where the condition 8 is not fulfilled, the solutions for $W_{1}$ and $W_{2}$ doesn't exist. This defines a set of loci, for a given position of one of the finger, where amplitudes can not be controlled, like the ones depicted on figure 1 for the position $x_{1}=0.25$ and $y_{1}=0.5$. This loci creates a limitation of the method, because the dual touch interaction cannot be obtained at any position. To avoid this limitation, one solution could be to use more than 2 modes. This solution has not been evaluated in this paper. 


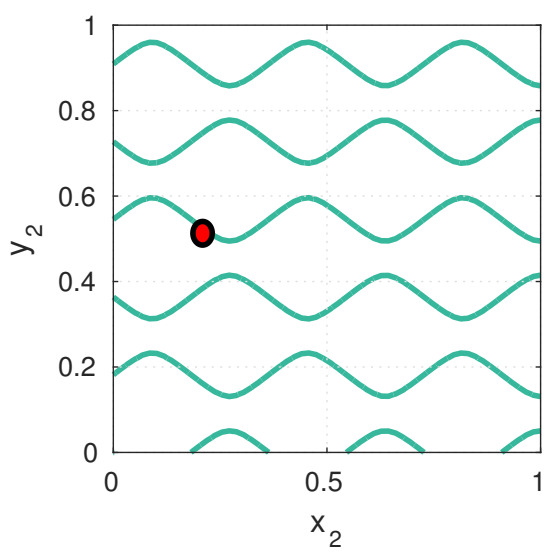

Fig. 1. Loci of the position of the second finger in the $x_{2}, y_{2}$ plane where amplitudes can not be set. These loci depends on the position of the first finger (symbolised by the ellipse)

\section{Validation in the $1 \mathrm{D}$ case}

\subsection{Experimental setup}

A $6 \times 6 \times 350 \mathrm{~mm}^{3}$ aluminium beam is actuated by two Langevin transducers. Two aluminium horns have been designed and manufactured in order to be able to transmit the vibration to the beam. The whole system is fixed on a rigid support, which is mobile to measure vibration velocity at several points. A laser interferometer (OFV-525/-5000-S) is used for that purpose. The control algorithm is implemented on a DSP (TI 2812) and two amplifiers (HSA 4051) supply power to the transducers.

For the experiment, the boundary condition is free-free; the two vibration modes 16 and 17 are selected with regards to the half-wavelength, which is approximately equal to $20,5 \mathrm{~mm}$. The working frequency is arbitrarily chosen between the resonant frequency of the two modes, that is $27,7 \mathrm{kHz}$.

A Linear Variable Differential Transformer (LVDT) is attached to the user's right hand index. By this way, we track its position and generate the references $\left[W_{M}\right]$ in the modal space. A closed loop control as described in [6] calculates the required voltage amplitude on each transducer. To simplify the set-up, the fingers are not tracked independently, but we considered that the second finger is located at a fixed position apart from the first one. In our experiment, we chose $3 \mathrm{~cm}$ as a convenient distance for the participants.

Figure 2-left shows the desired and measured vibration amplitude under each finger as a function of the position on the beam. To perform the measurements, the LVDT was manually moved and the amplitudes were measured at the planned positions. The systematic error observed is small (less than 0.1 
$\mu m$ ), and may by due to the errors in the identification of the deformation mode shape. Moreover, as it can be seen, it is actually possible to achieve in most cases the desired amplitudes, except at some points where the determinant of $\left[\Phi_{f}\right]$ is close to zero. In these cases, the actuator's voltage reference exceeds the maximum voltage amplifiers $(300 \mathrm{~V}$ peak to peak). Figure 2-right illustrates an example of the calculated and measured modal amplitude references as a function of planned positions of the two fingers on the beam.
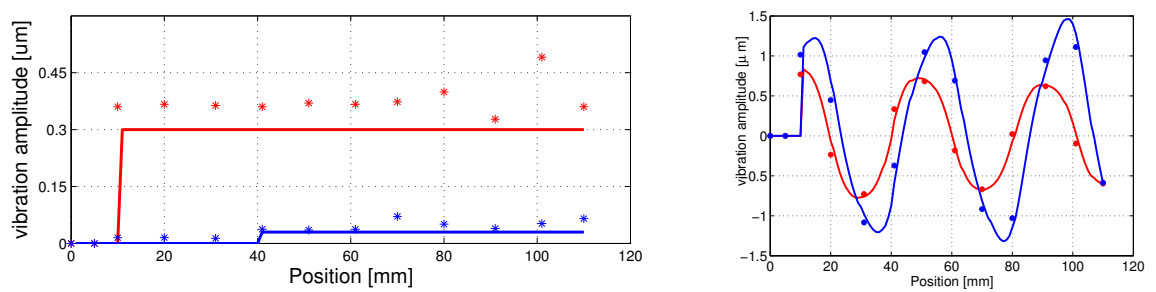

Fig. 2. Comparison between references $(-)$ and measurements $(*)$; left: vibration amplitude under the fingers as a function of the right finger (blue: left finger, red: right finger); right: modal amplitudes (blue: left actuator, red: right actuator

As it can be seen, the modal amplitudes and their references are in good agreements. However, a systematic error occurs on the vibration amplitude at the location of the two fingers. It has been observed a systematic lag of $1 \mathrm{~mm}$ on the nodes that may be attributed to an error in the identification of the modal shape $\phi_{n}$. However, the error remains under sensitivity threshold.

\subsection{Experimental Procedure and results}

Seven volunteers participated to the following experiment: 3 females, 4 males, all right handed, aged from 25 to 30 years. Two of them were familiar with VFD. Their hand was guided to explore the beam after washing. Three stimuli were presented. The first one was the beam without excitation. In the second one, the beam was activated with a standing wave of $1 \mu \mathrm{m}$, in order to create friction reduction. The subjects could explore the device with one finger. After this first part of the experiment, they were asked to qualify and to rate the friction reduction in their personnal scale.

In the second part of the experiment, the subjects could explore the beam using two fingers of left and right hands, separated by a constant distance. Here, the multi-finger control was activated to present an node of vibration under the right finger, and a vibration amplitude of approximately $1 \mu \mathrm{m}$ on the left one. They were then asked to rate the friction reduction on each fingers, according to their personal scale. The normalized results are summed up in figure 3. 


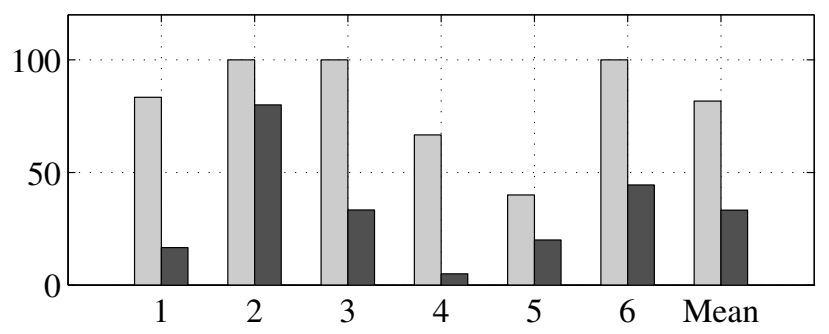

Fig. 3. Normalized perceived friction reduction for each participant (from 1 to 6 ) and average; light: left finger; dark: right finger.

\subsection{Discussion}

As a result of the experiment, the subjects felt the beam on the left finger more "slippery" or "smoother" than the friction on the right finger. This is consistent with the multifingers algorithm, which produced a node under the right finger and approximately $1 \mu \mathrm{m}$ of vibration under the left finger. Since the finger's position is tracked during the experiment, the node is sliding with user's finger. If a stationary wave would have been set on the beam, then the users would have felt nodes and antinodes of vibration successively on each fingers, and would not have detected a finger smoother than the other [4].

Moreover, 5 subjects found the beam more slippery for the left finger compared to the beam without any vibration. This highlights the fact that the plate vibrates on both side of the node. As a consequence, even though a node is always placed under the fingertip, there is still friction reduction. This reduction is however $48 \%$ (average, Standard deviation $24 \%$ ).

These results show that it is possible to create independent friction reduction under two fingertips, on a $1 D$ VFD, with an appropriate tracking of the finger, and the multi-finger algorithm of equation 5 . The next part of the paper presents the prototype for $2 D$ and 2-fingers VFD.

\section{Towards 2D}

\subsection{Design considerations}

In a first attempt to extend the previous study to the case of plate, a second setup has been realized. It was chosen to use two similar modes since in the case of square plates, two orthogonal modes with a same wavelength and theoretically a same frequency can be excited. In the case at hand, the $8^{t} h$ modes in the $X$ and $Y$ directions were selected based on the half-wavelength. Also, the working frequency should be above the audible spectrum, thus, the design was a $140 \times$ $140 \times 4 \mathrm{~mm}^{3}$ plate. This results in a rather thick plate as a consequence of the large required wavelength. Hence, this method may be more suitable for 


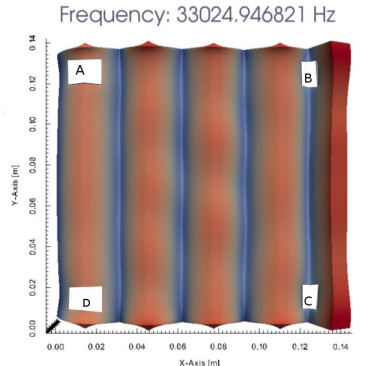

(a)

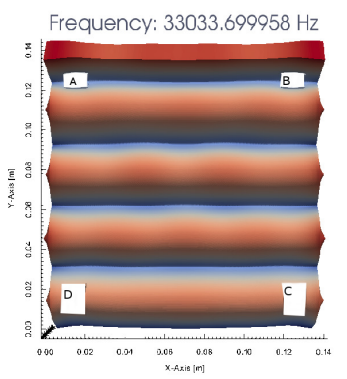

(b)

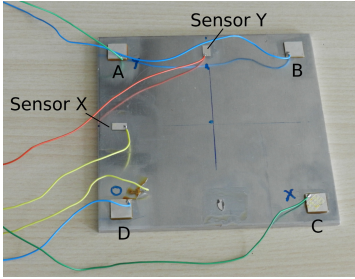

(c)

Fig. 4. Vibration modes and mode shapes using FEM with the position of the actuators (in white in a and b), and the prototype (c).

large tactile devices, where volume constraint should not be a problem.Figure 4 presents the plate and its actuators.

The placement of the actuators, which consists in piezoelectric patches glued on the surface of the plate, is also critical. Theoretically, two actuators would have been enough to excite the two modes. But to preserve the symmetry of the device, four actuators were used, denoted $A, B, C$ and $D$ in the sequel. Their location and the direction of their polarization is crucial for the application :

- $A$ and $D$ are placed on a antinode of vibration of mode $X$, while $B$ and $C$ are on an opposite antinode of vibration (figure 4, a)

- $C$ and $D$ are placed on a antinode of vibration of mode $Y$, while $A$ and $B$ are on an opposite antinode of vibration (figure $4, \mathrm{~b}$ )

- the two pairs of actuators $A$ and $D$ on one hand, and $C$ and $D$ on the other hand, are placed in such a way that their polarization direction are opposite.

These considerations have the following advantages:

- the four actuators have the same contribution to each mode, but can be additive or destructive,

- by connecting $A$ and $C$ to the same supply denoted $V_{A C}$, and $B$ and $D$ to $V_{B D}$, the contribution to mode $X$ is given by $V_{A C}+V_{B D}$, while the contribution to mode $Y$ is given by $V_{A C}-V_{B D}$. This allows to continuously mix both modes, and not to switch between them as in [9]

The device is supposed to work in closed loop, in order to control the amplitudes of the modes. Hence, in addition to the actuators, two aditionnal piezoelectric patches are used as sensors. In order to measure the actual amplitude of each mode, they have to be precisely placed. Ideally, they should be at the position of an antinode of one mode and a node of the other one [8].

\subsection{Experimental results}

Figure 5 shows the measured deflection as a function of frequency on the manufactured prototype. The two modes have similar resonant frequency $\left(f_{x}=33010\right.$ 
$\mathrm{Hz}, f_{y}=33064 \mathrm{~Hz}$ ), and the actuators have the same contributions since the amplitudes are similar.
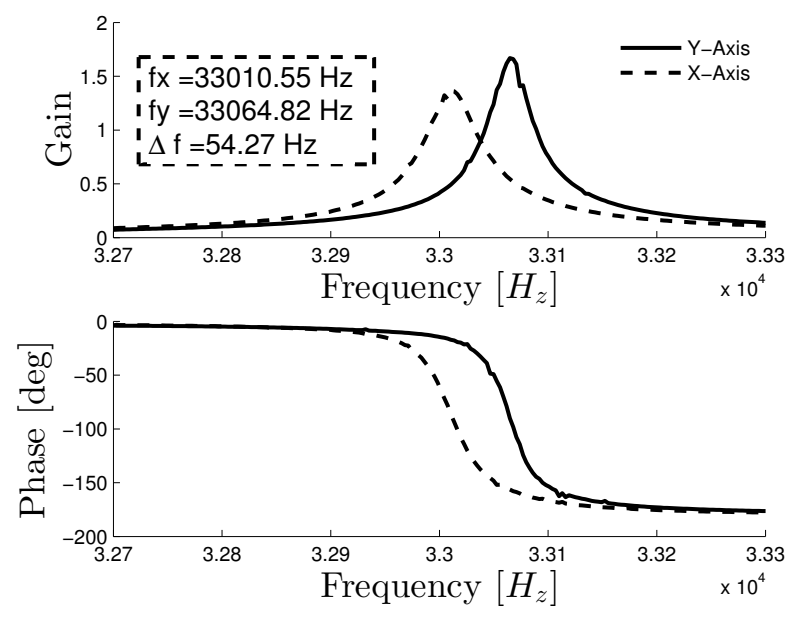

Fig. 5. Vibration amplitude of the 2 vibration modes as a function of frequency

We also show in figure 6 the deformation mode shapes of the two modes. They are close to the calculated one presented figure 4 .

The working frequency chosen for the experiment was equal to the median frequency of the modes. In this way, a mode is not promoted compared to the other one. However, as a consequence of the high quality factors of the resonances, the phase rotation is very stiff. It is thus necessary to adjust the voltages amplitude and phase, in order to compensate for the gain discrepancy and the phase shift.

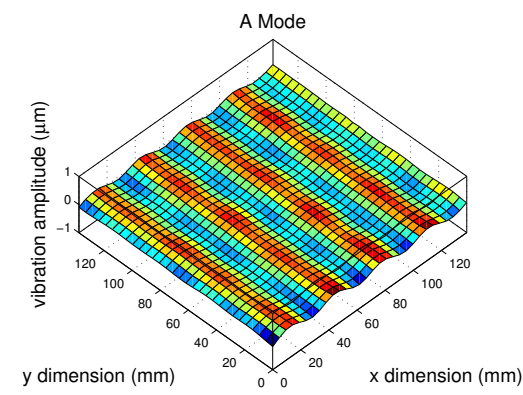

(a)

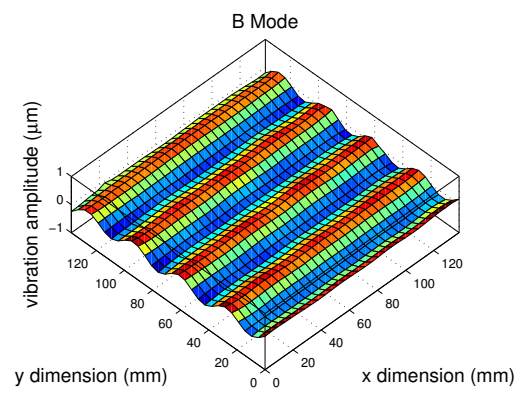

(b)

Fig. 6. Deformation mode shape; (a) $X$ mode, (b) $Y$ mode 
The figures 7 presents simulation and experimental measurements for different combinations of modes $X$ and $Y$ which confirm the possibility to control the spatial pattern of nodes and antinodes.

For example, the point $P_{1}$ in figure 7 is located on a node of vibration when $W_{X}=W_{Y}$, and becomes an antidone of vibration when $W_{X}=-W_{Y}$. Conversely, $P_{2}$ is an antinodde of vibration for the first case and a node of vibration for the second one.
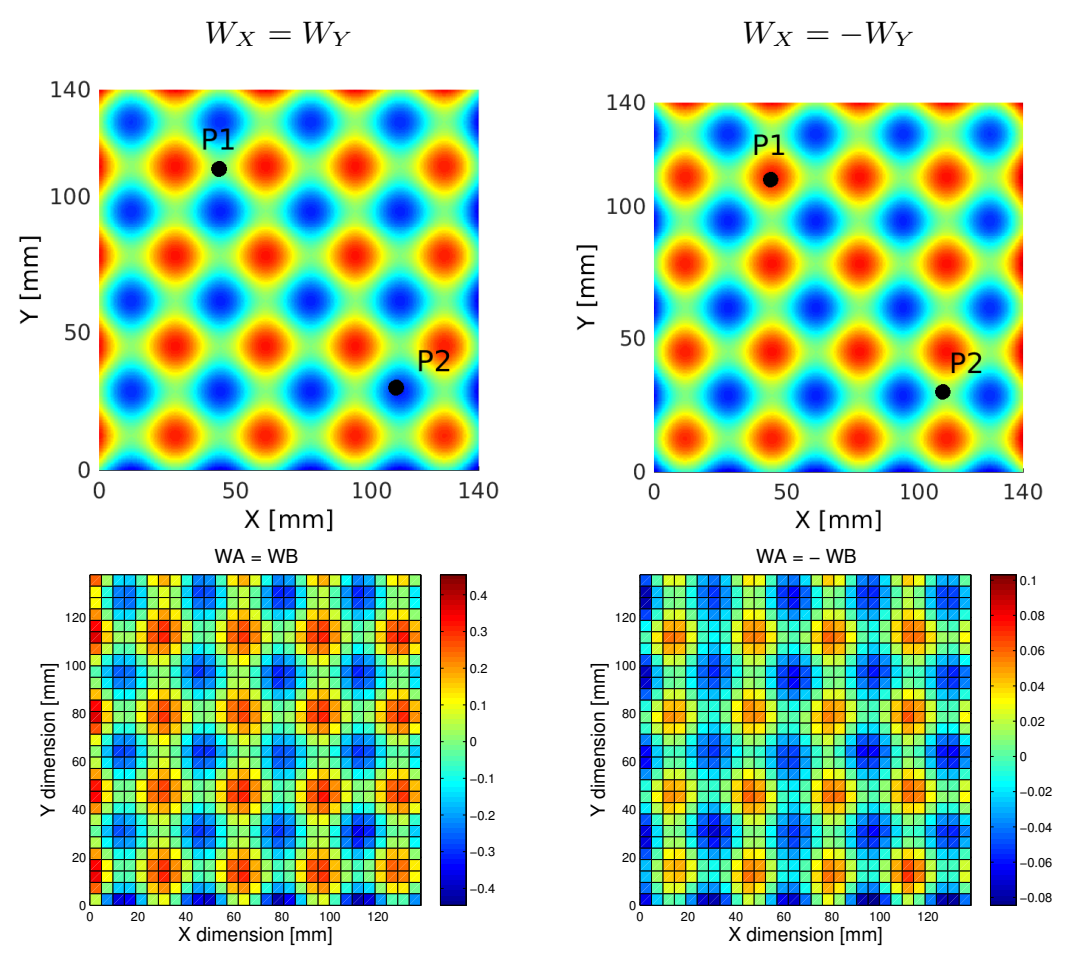

Fig. 7. Plate's deformation for several combinations modes $X$ and $Y$; top: simulation, bottom: experimental measurement.

\section{Conclusion}

This paper presents a Variable Friction Device which can independently control the friction reduction under two fingertips. First, the approach has been validated on a $1 D$ device. During the experiment, a beam was controlled in such a way that a node was continuously presented under a fingertip, while a second finger was moving over an antinode of vibration. Subjects have found that the sliding node is $48 \%$ less slippery than the sliding antinode of vibration. We then present 
the design of a $2 D$ device. The vibration control of the $X$ mode according to the $Y$ one allows to change smoothly and continuously the position of nodes and antinodes of vibration.

Future work will be dedicated to the introduce the tracking of the two fingers on the $2 D$ device. Moreover, introduction of a third vibration mode may be necessary in order to be able to control the friction over the whole display.

\section{Acknowledgement}

This work has been carried out within the framework of the project StimTac of IRCICA (institut de recherche sur les composants logiciels et matériels pour la communication avancée), and the Mint project of Inria.

\section{References}

1. Adams, M.J., Johnson, S.A., Lefèvre, P., Lévesque, V., Hayward, V., André, T., Thonnard, J.L.: Finger pad friction and its role in grip and touch. Journal of The Royal Society Interface 10(80) (2012)

2. Bau, O., Poupyrev, I., Israr, A., Harrison, C.: Teslatouch: Electrovibration for touch surfaces. In: Proceedings of the 23Nd Annual ACM Symposium on User Interface Software and Technology. pp. 283-292. UIST '10 (2010)

3. Chubb, E., Colgate, J., Peshkin, M.: Shiverpad: A glass haptic surface that produces shear force on a bare finger. Haptics, IEEE Transactions on 3(3), 189-198 (July 2010)

4. Ghenna, S., Giraud, F., Giraud-Audine, C., Amberg, M., Lemaire-Semail, B.: Preliminary design of a multi-touch ultrasonic tactile stimulator. In: World Haptics Conference (WHC), 2015 IEEE. pp. 31-36 (June 2015)

5. Giraud, F., Amberg, M., Lemaire-Semail, B., Casiez, G.: Design of a transparent tactile stimulator. In: Haptics Symposium (HAPTICS), 2012 IEEE. pp. 485-489 (March 2012)

6. Giraud, F., Giraud-Audine, C., Amberg, M., Lemaire-Semail, B.: Vector control method applied to a traveling wave in a finite beam. Ultrasonics, Ferroelectrics, and Frequency Control, IEEE Transactions on 61(1), 147-158 (January 2014)

7. K.F., G.: Wave Motion in Elastic Solids. Dover Publications (1991)

8. Nadal, C., Giraud-Audine, C., Giraud, F., Amberg, M., Lemaire-Semail, B.: Modelling of a beam excited by piezoelectric actuators in view of tactile applications. Mathematics and Computers in Simulation pp. - (2015)

9. Son, K.J., Kim, K.: The use of degenerate mode shapes in piezoelectric variablefriction tactile displays(the 12 th international conference on motion and vibration control). Dynamics and Design Conference 2014(12), 1-7 (aug 2014)

10. Vezzoli, E., Ben Messaoud, W., Amberg, M., Giraud, F., Lemaire-Semail, B., Bueno, M.A.: Physical and perceptual independence of ultrasonic vibration and electrovibration for friction modulation. Haptics, IEEE Transactions on 8(2), 235239 (April 2015)

11. Wiertlewski, M., Colgate, J.E.: Power optimization of ultrasonic frictionmodulation tactile interfaces. Transactions on Haptics 8(1), 43-53 (2014) 